\title{
Colour Evolution of Rosé Wines after Bottling
}

\author{
B. Hernández, C. Sáenz*, C. Alberdi, S. Alfonso and J.M. Diñeiro \\ Departamento de Física, Universidad Pública de Navarra, Campus Arrosadía, 31006 Pamplona, Navarra (SPAIN)
}

Submitted for publication: July 2010

Accepted for publication: September 2010

Key words: Colour appearance, wine colour, rosé wine, colour measurement, colour evolution

\begin{abstract}
This research reports on the colour evolution of six rosé wines during sixteen months of storage in the bottle. Colour changes were determined in terms of CIELAB colour parameters and in terms of the common colour categories used in visual assessment. The colour measurement method reproduces the visual assessment conditions during wine tasting with respect to wine sampler, illuminating source, observing background and sample-observer geometry. CIELAB $L^{*}, a^{*}, b^{*}, C^{*}$ and $h_{a b}$ colour coordinates were determined at seven different times ( $t=0,20,80,153,217,300$ and 473 days). The time evolution of colour coordinate values was studied using models related to linear, quadratic and exponential rise to a maximum. Adjusted $\mathbf{R}^{2}$, average standard error and CIELAB $\Delta \mathbf{E}^{*}$ colour difference were used to compare models and evaluate their performance. For each colour coordinate, the accuracy of model predictions was similar to the standard deviation associated with a single measurement. An average $\Delta E^{*}=0.92$ with a 90 percentile value $\Delta E^{*}{ }_{90 \%}=1.50$ was obtained between measured and predicted colour. These values are smaller than human colour discrimination thresholds. The classification into colour categories at different times depends on the wine sample. It was found that all wines take three to four months to change from raspberry to strawberry colour and seven to eight months to reach the redcurrant category. Only two wines had developed salmon colour by the end of the experiment.
\end{abstract}

\section{INTRODUCTION}

Colour is the most important visual attribute of wines and is an important factor in the overall quality of this product. Wine tasting starts with the assessment of a wine's colour and progresses to the evaluation of the other sensory attributes, like odour and in-mouth sensations (Jackson, 2002). With colour being the first assessed attribute, this assessment can even affect later quality and taste perceptions (Maga, 1974; Williams et al., 1984; Parr et al., 2003). As in other types of wine, the colour of rosé wine is an important factor affecting purchasing decisions. Both experienced and inexperienced subjects have shown definite and similar colour preferences regarding the colour of this particular product (Ough \& Amerine, 1967). With some exceptions, sparkling wines, for instance, the tasting process requires pouring the wine inside a standard tasting sampler (O.I.V., 1978; Jackson, 2002). The wine taster then assesses the colour of the wine while holding the tasting sampler in front of a white background and tilting the glass approximately $45^{\circ}$. In this way the wine surface takes on an oval shape inside the tasting glass and the depth of the wine along the surface varies from approximately $30 \mathrm{~mm}$ in the centre of the oval to negligible values near the border, called the rim. At each point of the surface, the variations in wine depth modulate the relative importance of the reflected, scattered and transmitted light through the wine. This creates a complete range of colour nuances that can be distinguished and evaluated by the taster when looking directly through the glass. Daylight is recommended for wine colour evaluation but artificial illumination resembling the characteristics of daylight is also appropriate. Wine products can be found in a great variety of colours and the taster's judgment aptitudes may depend on the type of wine (Gawel \& Godden, 2008).

The colour of liquids can be also determined by means of instruments. The determination of the optical properties of wines in the visible range traditionally has been obtained from transmittance and absorbance measurements. These measurements require the wine samples to be poured into standard plastic or quartz cells. The cell's optical path length may vary between 1 and $2 \mathrm{~mm}$ in the case of highly absorbing or scattering samples like red wines to 10 , or even $20 \mathrm{~mm}$, for highly transparent samples like white wines. Sample preparation usually requires filtering of the wine to reduce both the presence of small particles and unwanted light scattering during measurement (O.I.V., 1978). Transmittance measurements provide valuable information because absorbance depends on the concentration of pigments (Jackson, 1994; Alcalde-Eon et al., 2006; Versari et al., 2008). With respect to the colour characterisation of wines, various colour indices and colour descriptors have been proposed. These indices are obtained from absorbance measurements at a reduced number of wavelengths. The so-called Glories parameters (Glories, 1984) are obtained from absorbance values at 420,520 and $620 \mathrm{~nm}$ : Colour density $\left(A_{420}+A_{520}\right)$, colour tint $\left(A_{420} / A_{520}\right)$, colour intensity (CI = $\left.A_{420}+A_{520}+A_{620}\right)$ and percentage of yellow $\left(100 \times A_{420} / \mathrm{CI}\right)$, red $\left(100 \times A_{520} / \mathrm{CI}\right)$ and blue $\left(100 \times A_{620} / \mathrm{CI}\right)$. Selected wavelengths have been also used to quantify colour changes in white wines. Absorbance, in particular at $420 \mathrm{~nm}\left(\mathrm{~A}_{420}\right)$, has been used as a browning indicator (Peng et al., 1998). 
Colour stimuli are properly described in terms of a colour space, being colour coordinates a function of the spectral properties of the sample, and not a function of a single wavelength or a reduced set of wavelengths, regardless whether it is assessed from purely reflected or transmitted light or a combination of both (Wyszecki \& Stiles, 2000; CIE, 2004). In the last decade, CIELAB (CIE, 2004) colour coordinates obtained from transmission measurements have been used in several studies with regard to wine colour and colour stability (Skouroumounis et al., 2003, 2005; Recamales et al., 2006; Gomez-Miguez et al., 2007).

Colour parameters obtained from transmission/absorbance measurements are objective measurements of the optical properties of wines and are therefore useful in analysing the effect of additives and treatments. On the other hand, the ultimate interest in colour parameters is the determination of the colour appearance of the product. Several studies have combined transmittance or absorbance measurements and visual colour assessment and have shown a general lack of agreement and poor correlation between them (Little, 1980; Huertas et al., 2003; Bartowsky et al., 2004; Pridmore et al., 2005; Skouroumounis et al., 2005). Inconsistencies can be also observed in reported CIELAB values obtained from transmittance measurements. For some samples, lightness $\mathrm{L}^{*}$ values very close to 100 and even higher than this value have been reported in some studies (Skouroumounis et al., 2005; Recamales et al., 2006; GomezMiguez et al., 2007). The lightness coordinate L* must be constrained by definition between the values 0 and 100 . Very transparent samples, like white wines, are almost as transparent as the calibration target and, in this situation, measurement errors and calibration uncertainties can result in $\mathrm{L}^{*}$ values greater than 100. This problem also affects absorbance or transmittance values at particular wavelengths. Furthermore, due to the shape of the CIELAB colour solid that stretches near $\mathrm{L}^{*}=0$ and $\mathrm{L}^{*}=$ $100, \mathrm{~L}^{*}$ values close to 100 imply that the values of chromatic coordinates $\mathrm{a}^{*}$ and $\mathrm{b}$ * and chroma $\mathrm{C}^{*}$ must necessarily be small. Unless the relative errors in the measurements are reduced to the same factor, possible differences between samples could be masked by this fact.

The main reason behind this mismatch is that transmittance or absorbance colour and visual colour assessment by wine tasters are carried out under very different conditions regarding the observer, background, viewing geometry and sample preparation. The geometrical arrangement of observer, object and light source has a strong influence in the visually observed and instrumentally measured colour (Wyszecki \& Stiles, 2000). It has been shown that objective colour measurements expressed as CIELAB colour coordinates can reproduce the visual colour assessment of expert wine tasters if colour measurements are performed under the same conditions as visual tasting. The use of standardised wine samplers, daylight illumination, white observing background and a similar sampler-observer relative geometry is required. Under such conditions, instrumental colour measurements were able to predict the colour classification into colour categories determined by wine tasters. Classification scores better than $85 \%$ were obtained for red wines (Hernandez, Saenz et al., 2009) and even better scores for white and rosé wines (Saenz Gamasa et al., 2009). Red, rosé and white wines are types of wine with different colour and spectral characteristics and thus the success of the measurement method relies on the careful replication of the above conditions (Hernández et al., 2008). The reported measurement precision was comparable to the precision obtained in transmittance geometries. This suggested that the new method could be used for the same purposes as the transmittance methods, while having the advantage of giving results that are highly correlated with visual wine colour assessment.

In order to test this possibility, we studied the colour evolution of rosé wines after bottling in this investigation. During the elaboration of this type of wine, and due to the lack of a maceration stage, only a reduced amount of anthocyanins was extracted from the grape skins. However, the lack of stable anthocyanin-phenolic complexes and the predominance of monomeric anthocyanins result in colour and aroma instability (Ribéreau-Gayon, 2000). The quality of this product therefore slowly decreases with time once it has been bottled. In consequence, rosé wines preferably have to be consumed in the first year after elaboration.

Rosé wines typically are served cooled at 6 to $10^{\circ} \mathrm{C}$ and consumed during spring and summer. Even though time spent inside the bottle before consumption is less than one year, sensory attributes, including colour, change during those months. Qualitative colour changes are well known to wine experts, but very few studies that provide quantitative measurements have been undertaken. Recently, though, the effect of pre-fermentative maceration and enzymatic treatments in colour, polyphenol and aroma compounds in rosé wines has been studied (Salinas et al., 2003). Four different treatments and a control sample were used. While focusing on colour stability, transmittance measurements were used to compute the Glories colour index CI as well as CIELAB coordinates immediately before bottling and after three and six months inside the bottle. These authors mention that wine colour changes from "intense violet" to "salmon hue" in Monastrell (Mourvedre) rosé wines within a few months. Although this assertion is correct in terms of visual assessment, it can hardly be related to the quantitative data. Their data show some of the problems related to transmittance measurements, like very high lightness (L* $\approx 95$ ) which does not correspond to the visual experience for rosé wines. In the control sample hue $\mathrm{h}_{\mathrm{ab}}$ effectively increased, as expected. However, there are no statistically significant differences in $\mathrm{a}^{*}$ between measurements and hue change comes solely from the variation of the $b^{*}$ coordinate. On the contrary, when measurements are performed in visual tasting conditions, both coordinates $a^{*}$ and $b^{*}$ contribute to the change in hue values. (Hernández et al., 2008; Saenz Gamasa et al., 2009).

Measuring colour at two or three different times informs us about the effect of treatments and additives in colour stability. In this context, a detailed picture of the colour evolution inside the bottle will be of great interest. Obtaining a qualitative and quantitative description of this evolution is therefore the main objective of the present study. As a second objective we will show that colour can be accurately measured under the same conditions as those under which visual tasting is carried out. Measurement accuracy will allow different time evolution models to be fitted and compared for each colour coordinate and sample. We will also provide the colour evolution of rosé wines in terms of the traditional colour categories used to classify rosé wine colour. 


\section{MATERIALS AND METHODS \\ Rosé wine samples}

Six different commercial rosé wines from six different wineries were studied. One rosé wine belonged to the Appellation of Origin "Cariñena" and the other five wines to the Appellation of Origin "Navarra". There were varietals made of Garnacha (2), Merlot (1) and coupages of Cabernet-Tempranillo (1), Cabernet-Garnacha (1) and Cabernet-Tempranillo-Merlot (1). Seven bottles of each wine were purchased directly from the wineries to guarantee that, for each wine, all were bottled at the same time. Throughout this work samples are labelled s1 to s6. Natural cork was used as closure in samples s1, s5 and s6; the other samples used synthetic closures.

\section{Colour measurements}

Wine was poured into normalised wine samplers (O.I.V., 1978) immediately after uncorking the bottle to prevent colour changes due to oxidation. The volume of liquid was carefully controlled to ensure that wine samplers were filled exactly to $1 / 3$ of their capacity $(75 \mathrm{ml})$. Once filled with wine, the sampler was placed inside a light booth - Gretag Macbeth Judge II - that provided the daylight illumination simulating the D65 standard illuminant. The sampler was held at a $45^{\circ}$ angle with respect to the horizontal, using an especially designed sampler holder. The colour of the walls and the floor of the light booth corresponded to a neutral grey with an approximate reflectance of $50 \%$, which is the customary setting in these devices. Therefore, to provide the desired white background during the measurements, the floor of the light booth was covered with a white diffuse screen.

When the wine sampler is tilted, as mentioned above, the wine surface takes on an oval shape. Wine depth varies throughout the observed surface, producing a continuous colour variation over it. The region we call the centre is located in the central part of the oval. Wine depth is about $30 \mathrm{~mm}$ in this region and the colour is rather uniform. The outer part of the oval, defined by its perimeter, is called the rim. In this region, wine depth and colour varies from one point to another. The optimal measurement region depends on the type of wine. For red wines, measurements in the rim are preferred (Hernández et $a l ., 2008)$. For rosé and white wines, the best results are obtained in the centre region (Saenz Gamasa et al., 2009). In the present study, measurements accordingly were performed in the centre region. For each wine sample, three independent measurements were taken by pouring the wine into three separate samplers. Two readings were taken in each sampler and each wine sample was therefore measured six times.

Spectral radiance measurements were recorded with a Photo Research PR650 spectroradiometer in the range 380 to $780 \mathrm{~nm}$ at $4 \mathrm{~nm}$ intervals. Measurements were taken directly through the glass sampler at an angle of $45^{\circ}$ with respect to the wine surface,. A lambertian white diffuser was used to measure the illumination and calibrate the system. The CIELAB 1976 colour coordinates $L^{*}, a^{*}, b^{*}, C^{*}$ and $h_{a b}$ were computed, using the D65 standard illuminant and CIE $19312^{\circ}$ standard observer (CIE, 2004). In the CIELAB 1976 colour space $L^{*}$ is the approximate correlate of the perceived attribute of lightness. Coordinate $a^{*}$ records positive values for reddish colours and negative values for greenish colours. Coordinate $b^{*}$ records positive values for yellowish colours and negative values for bluish colours. $L^{*}, a^{*}$ and $b^{*}$ form a rectangular coordinate system in a three-dimensional, approximately uniform colour space. Any point in this colour space can be also defined by the cylindrical coordinates $L^{*}, C^{*}$ and $h_{a b}$. The $C^{*}$ and $h_{a b}$ coordinates are the approximate correlates with the perceived attributes of chroma and hue respectively and they are the polar coordinates in the $a^{*} b^{*}$ plane defined by:

$$
\begin{gathered}
C^{*}=\sqrt{\left(a^{*}\right)^{2}+\left(b^{*}\right)^{2}} \\
h_{a b}=\arctan \left(\frac{b^{*}}{a^{*}}\right)
\end{gathered}
$$

In the CIELAB 1976 colour space colour differences $\Delta E_{a b}^{*}$ between two colour stimuli can be calculated using the Euclidean distance defined by:

$$
\Delta E_{a b}^{*}=\sqrt{\left(\Delta L^{*}\right)^{2}+\left(\Delta a^{*}\right)^{2}+\left(\Delta b^{*}\right)^{2}}
$$

where $\Delta L^{*}, \Delta a^{*}$ and $\Delta b^{*}$ are the differences in the values of each colour coordinate between the two stimuli.

\section{The measurement schedule}

Colour measurements were performed at different dates. On each measurement day a new bottle of each wine was uncorked and the wine measured as described in the previous section. The first measurement date was in April 2008. We will consider this date as the beginning of our time scale or $t=0$. The rest of the measurement dates correspond to $t=20,80,153,217,300$ and 473 days after the first measurement. Thus measurements approximately covered the colour evolution of rosé wines within the bottle over 16 months. Wines were kept in the dark and at room temperature $\left(20^{\circ} \mathrm{C}\right)$ until they were measured, resembling the storage conditions in wine shops and supermarkets. Wine samples were discarded after each measurement.

\section{Data analysis}

Modelling the colour evolution of rosé wines inside the bottle was one of the objectives of the present study. Three different parametric models having explicit time dependence were used. First a simple linear model (LIN) of the form:

$$
y=y_{0}+y_{1} \cdot t
$$

where $y$ represents any colour coordinate and $\mathrm{y}_{0}$ and $\mathrm{y}_{1}$ are the model free parameters to be determined after fitting the model to the experimental data.

Some colour coordinates exhibit a clear nonlinear dependence over time. In order to account for this nonlinear dependence we have used a quadratic model (QUAD) and an exponential rise to a maximum model (ERM) which are described by the following expressions:

$$
\begin{gathered}
y=y_{0}+y_{1} \cdot t+y_{2} \cdot t^{2} \\
y=y_{0}+y_{1} \cdot\left[1-\exp \left(-y_{2} \cdot t\right)\right]
\end{gathered}
$$

As before, $y$ represents any colour coordinate and $\mathrm{y}_{0}, \mathrm{y}_{1}$ and $\mathrm{y}_{2}$ are the model parameters to be determined. The LIN model has two free parameters and the QUAD and the ERM models have three free parameters each. The QUAD model is the straightforward 
extension of the LIN model including a nonlinear term. The ERM model is what we should expect if colour coordinate values changed according to a first order kinetics reaction. In all models, the variable $t$ is the time since the first measurement expressed in days. Notice that $\mathrm{t}=0$ corresponds to the first measurement day and not to the moment when the wine was bottled.

The goodness of fit for each model and each colour coordinate was evaluated using the adjusted $\mathrm{R}^{2}$ values and the standard error (S.E.) of the model predictions with respect to the actual experimental values. The $\mathrm{R}^{2}$ statistic is known to weakly increase with the number of parameters in the model. Adjusted $\mathrm{R}^{2}$ is a modification of $\mathrm{R}^{2}$ that adjusts for the number of parameters in the model. Since we have models with two and three parameters, the adjusted $\mathrm{R}^{2}$ has been used in order to compare them. In any case, adjusted $\mathrm{R}^{2}$ is always less or equal to $\mathrm{R}^{2}$.

\section{RESULTS AND DISCUSSION}

Wineries and grape varieties that provided the studied samples varied, but all wines were elaborated and commercialised at similar dates. Their respective tasting cards, which were filled in just before commercialisation, catalogued all samples as having raspberry colour. Although catalogued in the same visual colour category, all samples differed with regard to the instrumentally measured colour. Table 1 summarises the CIELAB colour coordinates of each wine sample at $t=20$ days as well as the results of the ANOVA and Tukey tests performed to evaluate the differences among samples. Colour coordinate values and measurement errors were in the range of values reported in our previous work (Saenz Gamasa et al., 2009). Considering L* a* and $b^{*}$, there are significant differences $(p<0.05)$ among all wine samples in at least one colour coordinate. Most frequently differences among samples affect two colour coordinates. The same situation is found if we consider $L^{*}, C^{*}$ and $h_{a b}$. In fact, $h_{a b}$ alone discriminated among most of the studied samples. From this point of view, all samples had different colour. Despite the evolution of colour coordinates over time, the same situation was found at all measurement times.

The measurement precision in each colour coordinate was evaluated using the standard deviation associated with the six measurements performed on each wine sample at each measurement time. It was found that the standard deviation had similar values regardless of the wine sample or measurement time. Therefore the average standard deviation over all samples and measurement times was an adequate measure of the overall measurement precision. These values are listed in the last row of Table 1.

Rosé wine colour evolves over time inside the bottle. In Fig. 1 we show the mean values of each colour coordinate as a function of the time $t$ for each rosé wine sample. Visual inspection of these figures evidences the distinctive characteristics of the time evolution of each colour coordinate. If we consider a particular colour coordinate, all studied samples exhibit similar qualitative behaviour. However, differences among samples can be also noticed and the quantitative analysis was carried out separately for each colour coordinate and each wine sample. For each colour coordinate and sample, the time dependence was fitted to the three models LIN, QUAD and ERM. The adjusted $\mathrm{R}^{2}$ values and standard errors (S.E.) of the predicted values are summarised in Table 2 . In some cases, the attempts to fit the observed values to QUAD and ERM models did not produce satisfactory results. In these cases, the non-linear model parameters $\left(\mathrm{y}_{1}, \mathrm{y}_{2}\right.$ or both) were compatible with zero and were not statistically significant with large associated $\mathrm{p}$-values $(\mathrm{p}>0.1)$. When this happened, models were marked as not significant (ns), as seen in Table 2. The best fitting result for each colour coordinate and sample has been highlighted in bold face. In Fig. 2 an example of the fitting functions to the experimental data is shown.

On average, lightness $\mathrm{L}^{*}$ was uncorrelated with time $\left(\mathrm{R}^{2}\right.$ $<0.01)$ and remained constant during the studied period of time. Considering each sample separately, there were three (s2, s3 and s6) with $L^{*}$ values of $42.67 \pm 0.28,45.58 \pm 0.29$ and $42.89 \pm 0.46$ respectively that were statistically constant over time $(\mathrm{p}<0.05)$. In these cases the LIN model was marked as not significant in Table 2. On the other hand, there was one sample (s1) with an $\mathrm{L}^{*}$ value that slowly increased at an average rate of $0.010 \pm 0.002$ day $^{-1}$. This sample was best fitted using the QUAD model with a S.E. value of 0.77 . There also were two samples (s4 and s5) whose $L^{*}$ values decreased at $-0.014 \pm 0.003$ per day and $-0.006 \pm 0.002$ per day and were well described by the LIN model. As a result, the range of observed $L^{*}$ values spread from 41 to 46 at $\mathrm{t}=0$ to 33 to 48 after 16 months. The standard error of the predicted $\mathrm{L}^{*}$ values was less than 1.30 units in all cases.

During storage inside the bottle, the red-green colour coordinate $a^{*}$ steadily decreased in all samples. The linear relationship between $a^{*}$ and $t$ suggested by the figure was confirmed by the good results obtained with the LIN model.

\section{TABLE 1}

Sample CIELAB colour coordinates at $\mathrm{t}=20$ days. Mean values and standard error $(\mathrm{n}=6)$ for each colour coordinate are shown. Different superscripts in the same column mean that differences are statistically significant at $\mathrm{p}<0.05$. The average standard deviation (SD) and its standard error in the determination of each colour coordinate are shown in the last row.

\begin{tabular}{|c|c|c|c|c|c|}
\hline Sample & $\mathrm{L}^{*}$ & $\mathrm{a}^{*}$ & $\mathrm{~b}^{*}$ & $\mathrm{C}^{*}$ & $\mathrm{~h}_{\mathrm{ab}}$ \\
\hline s1 & $42.5 \pm 0.2^{b}$ & $51.9 \pm 0.3^{\mathrm{c}}$ & $24.8 \pm 0.2^{\mathrm{bc}}$ & $57.5 \pm 0.4^{\mathrm{c}}$ & $25.5 \pm 0.1^{\mathrm{ab}}$ \\
\hline s2 & $42.0 \pm 0.6^{b}$ & $49.8 \pm 0.2^{b}$ & $23.1 \pm 0.5^{\mathrm{a}}$ & $54.9 \pm 0.4^{\mathrm{ab}}$ & $24.8 \pm 0.4^{\mathrm{a}}$ \\
\hline s3 & $44.7 \pm 0.3^{c}$ & $49.8 \pm 0.2^{b}$ & $25.0 \pm 0.2^{b c}$ & $55.7 \pm 0.3^{b}$ & $26.6 \pm 0.1^{\mathrm{c}}$ \\
\hline s4 & $38.6 \pm 0.4^{\mathrm{a}}$ & $50.7 \pm 0.3^{b}$ & $27.8 \pm 0.3^{\mathrm{d}}$ & $57.8 \pm 0.4^{\mathrm{c}}$ & $28.7 \pm 0.2^{\mathrm{e}}$ \\
\hline s5 & $43.0 \pm 0.3^{b}$ & $48.3 \pm 0.2^{\mathrm{a}}$ & $23.9 \pm 0.3^{a b}$ & $54.0 \pm 0.2^{\mathrm{a}}$ & $26.2 \pm 0.2^{b c}$ \\
\hline s6 & $45.0 \pm 0.2^{c}$ & $48.1 \pm 0.2^{\mathrm{a}}$ & $25.3 \pm 0.2^{c}$ & $54.3 \pm 0.3^{\mathrm{ab}}$ & $27.8 \pm 0.2^{\mathrm{d}}$ \\
\hline SD & $0.6 \pm 0.3$ & $0.5 \pm 0.2$ & $0.6 \pm 0.2$ & $0.7 \pm 0.3$ & $0.3 \pm 0.1$ \\
\hline
\end{tabular}



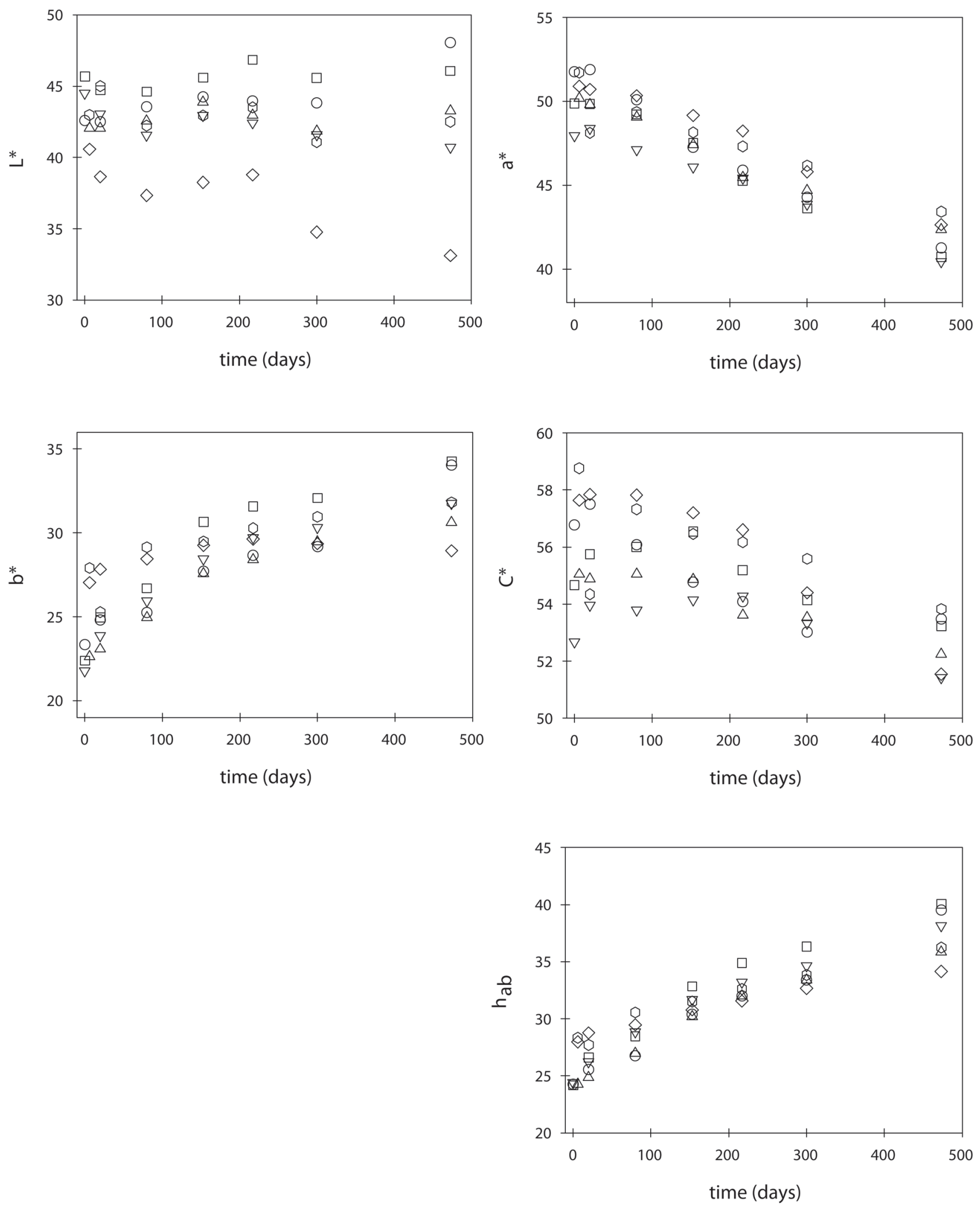

FIGURE 1

Evolution of the CIELAB L* $\mathrm{a}^{*}, \mathrm{~b}^{*}, \mathrm{C}^{*}$ and $\mathrm{h}_{\mathrm{ab}}$ colour coordinates with time for the six studied rosé wines. Time is measured with respect to the first measurement day $(\mathrm{t}=0)$. Each symbol corresponds to a different sample ( $\mathrm{s} 1$ : circles; $\mathrm{s} 2$ : up triangles; $\mathrm{s} 3$ : squares; s4: diamonds, s5: down triangles, s6: hexagons). 
Furthermore, the rate of change in $a^{*}$ was very similar in all samples and the dispersion in $a^{*}$ values remained invariable throughout the studied period of time. A linear fit over the entire sample set provided a good estimation of the decay rate in $a^{*}$ for an average rosé wine. Averaging over all samples, the CIELAB $a^{*}$ coordinate decreased at $-0.018 \pm 0.001$ per day equivalent to one unit every two months.

The good results obtained by a LIN fit can be slightly improved for some samples, including the nonlinear term of the QUAD model, both increasing the adjusted $\mathrm{R}^{2}$ and reducing the S.E. Taking the best model for each sample, the standard error S.E. of the predicted values was less than 0.5 units in all samples except for sample s6 $($ S.E. $=1.09)$. These values are comparable to the average standard deviation associated to the determination of the $\mathrm{a}^{*}$ coordinate in Table 1.

Unlike $a^{*}$, the nonlinear behaviour of $b^{*}$ over time inside bottle is evident in Fig. 1. In all cases $b^{*}$ increased with time and the increase rate in general was higher during the first six months. Five of the samples showed this behaviour, although there were differences in the increase rates. There was one sample whose $b^{*}$ coordinate decreased during the three last measurements. Except for sample s1, both $\mathrm{R}^{2}$ and S.E. were greatly reduced with the QUAD and ERM models with respect to the values obtained using the LIN fit. In general, ERM gave better results, although the differences between both nonlinear models were not large. Taking the best fitting function for each sample, the standard errors, except for sample s6, were less than 0.75 of a unit and comparable to the standard deviation associated to each colour measurement (Table 1).
Although the $\mathrm{C}^{*}$ coordinate was not constant, it decreased very slowly over time. The slow rate of change was due to the opposite behaviour of $\mathrm{a}^{*}$ and $\mathrm{b}^{*}$ over time and the definition of $\mathrm{C}^{*}$ (equation ). During the first six months, a* approximately decreased at the same rate as $b^{*}$ increased, resulting in an almost constant $\mathrm{C}^{*}$. As the increase rate of $\mathrm{b}^{*}$ slowed down, $\mathrm{C}^{*}$ started to decrease like $\mathrm{a}^{*}$. Nevertheless, the absolute change in $\mathrm{C}^{*}$ was small and on average took approximately one year to decrease by 2.5 units, which is less than $5 \%$ of its value. Except for sample s6, the errors associated with the predicted values were similar to the measurement dispersion in this coordinate.

We finally consider the CIELAB hue coordinate $h_{a b}$, which varies over time, like $b^{*}$. As in the case of $b^{*}$, sample s1 was well fitted using the linear model and, for this sample, the extension to nonlinear models did not improve the results. For the rest of the samples, QUAD and ERM models noticeably improved both the adjusted $\mathrm{R}^{2}$ and the S.E. values. In general, ERM gave the best results, although differences between the two models were not big. As with the rest of coordinates, standard errors in the predicted values were comparable to the standard deviations in single sample measurements.

In summary, measurement precision allows different evolution models to be tested and compared for each sample and colour coordinate. However, although individual colour coordinates can be accurately predicted by these models, two colour stimuli can not be compared using colour coordinates separately. Within the CIELAB framework the comparison between two colour stimuli is computed by means of the $\Delta \mathrm{E}^{*}$ colour difference value given by equation. Colour differences

TABLE 2

Adjusted $\mathrm{R}^{2}$ and Standard Error (S.E.) after fitting the time evolution of each colour wine sample. Three models were used: Linear model (LIN), quadratic model (QUAD) and exponential rise to a maximum (ERM). Best fitting results are highlighted in boldface.

\begin{tabular}{|c|c|c|c|c|c|c|c|c|c|c|c|}
\hline \multirow{2}{*}{\multicolumn{2}{|c|}{ Sample }} & \multicolumn{2}{|c|}{$\mathrm{L}^{*}$} & \multicolumn{2}{|c|}{$a^{*}$} & \multicolumn{2}{|c|}{$b^{*}$} & \multicolumn{2}{|c|}{$\mathrm{C}^{*}$} & \multicolumn{2}{|c|}{$\mathrm{h}_{\mathrm{ab}}$} \\
\hline & & $\mathrm{R}^{2}$ & S.E. & $\mathrm{R}^{2}$ & S.E. & $\mathrm{R}^{2}$ & S.E. & $\mathrm{R}^{2}$ & S.E. & $\mathrm{R}^{2}$ & S.E. \\
\hline \multirow[t]{3}{*}{$\mathrm{s} 1$} & LIN & 0.773 & 0.89 & 0.971 & 0.68 & 0.967 & 0.65 & 0.746 & 0.86 & 0.986 & 0.63 \\
\hline & $Q U A D$ & 0.829 & 0.77 & 0.990 & 0.40 & ns & ns & 0.924 & 0.47 & ns & ns \\
\hline & $E R M$ & ns & ns & 0.990 & 0.41 & ns & ns & 0.882 & 0.59 & ns & ns \\
\hline \multirow[t]{3}{*}{$\mathrm{s} 2$} & $L I N$ & $\mathrm{~ns}(*)$ & ns & 0.973 & 0.49 & 0.866 & 1.15 & 0.897 & 0.35 & 0.929 & 1.18 \\
\hline & $Q U A D$ & $\mathrm{~ns}$ & ns & 0.983 & 0.38 & 0.991 & 0.29 & ns & ns & 0.996 & 0.29 \\
\hline & $E R M$ & ns & $\mathrm{ns}$ & 0.983 & 0.39 & 0.993 & 0.26 & ns & $\mathrm{ns}$ & 0.995 & 0.30 \\
\hline \multirow[t]{3}{*}{ s3 } & $L I N$ & $\mathrm{~ns}(*)$ & ns & 0.980 & 0.50 & 0.822 & 1.82 & 0.391 & 0.90 & 0.926 & 1.56 \\
\hline & $Q U A D$ & ns & ns & Ns & ns & 0.952 & 0.95 & ns & ns & 0.983 & 0.73 \\
\hline & $E R M$ & $\mathrm{~ns}$ & $\mathrm{~ns}$ & Ns & ns & 0.970 & 0.75 & ns & ns & 0.986 & 0.67 \\
\hline \multirow[t]{3}{*}{ s4 } & $L I N$ & 0.745 & 1.30 & 0.973 & 0.50 & 0.326 & 0.77 & 0.897 & 0.76 & 0.967 & 0.40 \\
\hline & $Q U A D$ & ns & ns & 0.985 & 0.38 & 0.905 & 0.29 & 0.968 & 0.42 & 0.992 & 0.20 \\
\hline & $E R M$ & ns & ns & Ns & ns & 0.878 & 0.33 & ns & $\mathrm{ns}$ & 0.992 & 0.20 \\
\hline \multirow[t]{3}{*}{ s5 } & $L I N$ & 0.552 & 0.83 & 0.980 & 0.39 & 0.820 & 1.55 & 0.171 & 0.93 & 0.936 & 1.22 \\
\hline & $Q U A D$ & ns & ns & 0.990 & 0.27 & 0.972 & 0.62 & 0.826 & 0.43 & 0.986 & 0.58 \\
\hline & $E R M$ & ns & ns & Ns & ns & 0.989 & 0.38 & ns & $\mathrm{ns}$ & 0.991 & 0.45 \\
\hline \multirow[t]{3}{*}{ s6 } & $L I N$ & $\mathrm{~ns}(*)$ & ns & 0.820 & 1.09 & 0.683 & 1.22 & 0.247 & 1.47 & 0.951 & 0.67 \\
\hline & $Q U A D$ & ns & ns & Ns & ns & 0.717 & 1.15 & ns & ns & 0.969 & 0.53 \\
\hline & $E R M$ & ns & $\mathrm{ns}$ & Ns & ns & 0.722 & 1.14 & ns & ns & 0.971 & 0.51 \\
\hline
\end{tabular}

ns: not significant $(\mathrm{p}>0.1)$

(*) Data do not change significantly over time; mean value is the best data description. 
can be used to evaluate whether two colour stimuli can or can not be distinguished by a human observer. This requires knowledge of the colour discrimination thresholds, or minimum discernible $\Delta \mathrm{E}^{*}$ differences. These thresholds depend on the particular choice of samples, illumination and viewing conditions.

At present, we do not have threshold $\Delta \mathrm{E}^{*}$ values in the case of visual comparison of wine samples. In fact, threshold $\Delta \mathrm{E}^{*}$ values do not have precise meaning in the case of complex stimuli like the wine surface in a tilted wine taster. In complex stimuli, colour changes from one point to another and unique colour coordinates $\mathrm{L}^{*}, \mathrm{a}^{*}$ and $\mathrm{b}^{*}$ can not be defined for the entire sample. Therefore colour difference formulae can be only computed or assessed between simple stimuli, usually two plain colour samples, in paired comparison experiments and under tightly controlled illuminating and viewing conditions (Schanda, 2007). Nonetheless, comparison between similar points from two complex stimuli can still give us a valuable indication of the colour difference between them. A value of $\Delta \mathrm{E}^{*}=3$ units has been suggested as an absolute colour discrimination threshold for red wines (Martinez et al., 2001). However, this value was not obtained under the conditions in which visual colour tasting of wines is carried out. Furthermore, red and rosé wines have very different colours and CIELAB
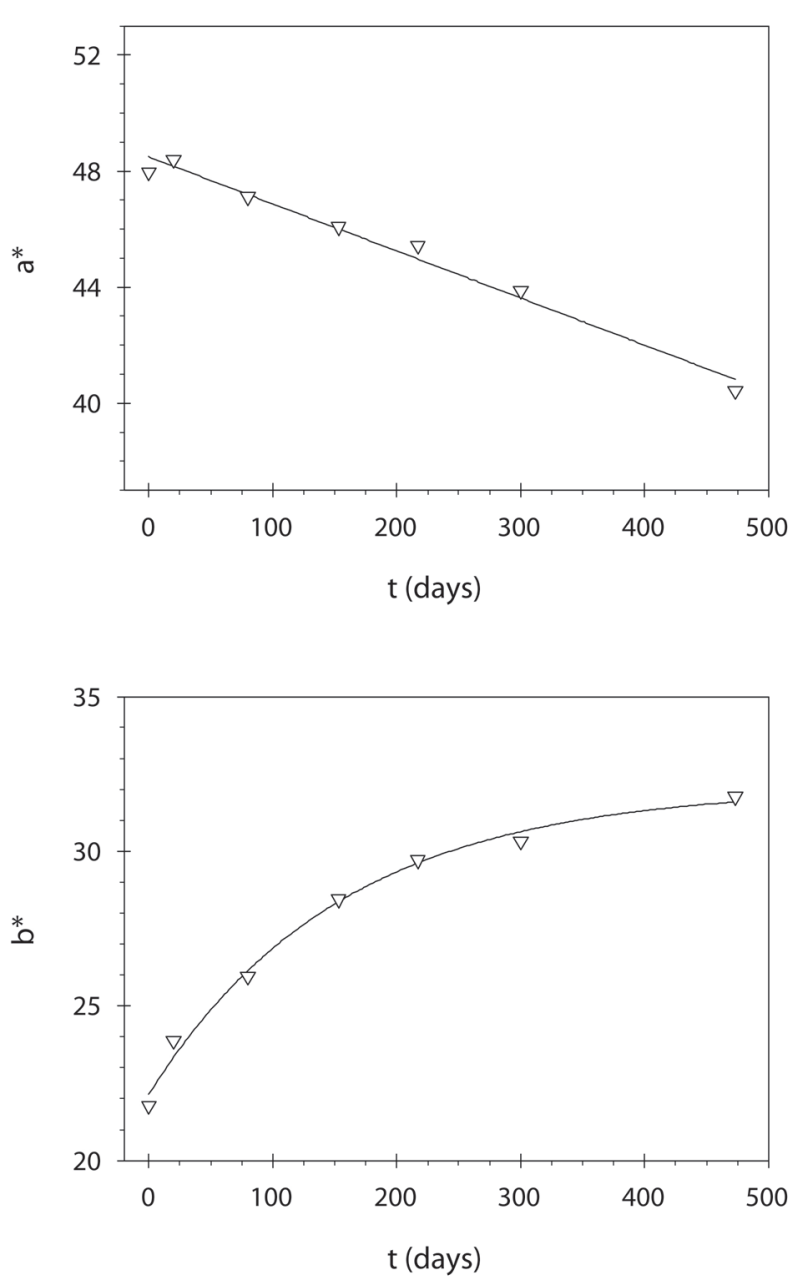

FIGURE 2

Measured values and best model predictions for the time evolution of $\mathrm{a}^{*}$ (top) and $\mathrm{b}^{*}$ (bottom) of sample s3. is known to be only approximately uniform. Experiments for wine colour classification into colour categories do not provide colour discrimination thresholds, since wines classified in the same colour category may be distinguished by colour if shown simultaneously (Hernandez et al., 2009; Saenz Gamasa et al., 2009). Recently, colour discrimination data from different sources have been reviewed in terms of present colour difference formulae and common colour spaces providing an average threshold value $\Delta \mathrm{E}^{*}=2.3 \pm 1.3$ (Mahy et al., 1994).

In order to evaluate the overall accuracy of the measurement method and the fitting models we have calculated the CIELAB colour differences $\Delta \mathrm{E}^{*}$ between the measured colour and the colour predicted by the fitting functions. CIELAB $\Delta \mathrm{E}^{*}$ colour differences were computed using equation 3 for each wine sample and each measurement day. In choosing the model functions two different cases were considered. First we used the predictions obtained with the LIN models for all colour coordinates. Although this model is not always the optimal choice, it is simple and has only two free parameters. We also used the best fitting function for each colour coordinate and sample. This second choice was expected to give the best colour estimation for each sample and measurement time.

Using the LIN models for all variables, an average $\Delta \mathrm{E}^{*}$ $=1.31$ units $($ standard deviation $=0.69)$ was obtained. Colour differences between measured and predicted values in general were less than 2.3 units with a $90 \%$ percentile $\Delta \mathrm{E}^{*}{ }_{90 \%}=$ 2.27. Although the LIN model is not always optimal colour differences were small and less than $\Delta \mathrm{E}^{*}=3$ units. Results improved when the best fitting functions were selected to predict each colour coordinate. In this case the average colour difference was $\Delta \mathrm{E}^{*}=0.92($ standard deviation $=0.62)$ and the $90 \%$ percentile was located at $\Delta \mathrm{E}^{*}{ }_{90 \%}=1.50$. Colour differences were approximately $30 \%$ smaller than in the LIN case.

The colour difference $\Delta \mathrm{E}^{*}=0.92\left(\right.$ or $\Delta \mathrm{E}^{*}=1.31$ ) was smaller than the colour difference thresholds obtained in the case of paired comparison experiments $\Delta \mathrm{E}^{*}=2.3 \pm 1.3$. This means that the colour evolution of rosé wines can be measured and modelled with the precision required for any application related to visual colour assessment of this kind of sample.

Taking advantage of the relationship between measured colour and visual assessment, we can use the colour evolution models to predict the colour category of each wine at any time within the time domain of the models. Fig. 3 shows the measured wine colour in the $\mathrm{a}^{*} \mathrm{~b}^{*}$ plane together with the prediction obtained by the best fitting functions for each coordinate. In order to determine the correspondence between $\mathrm{a}^{*} \mathrm{~b}^{*}$ values and colour categories, we used the discriminant scores of the discriminant functions obtained in an earlier study (Saenz Gamasa et al., 2009). The legitimacy of the discriminant functions was assumed here, since they were obtained from 33 samples of rosé wines showing similar characteristics with respect to origin, grape varieties and elaboration methods as those studied in this investigation. The approximate boundaries between colour categories are also given in Fig. 3. Since the discriminant functions depend on $\mathrm{L}^{*}$, a particular value of this coordinate must be chosen to plot the boundaries in the $\mathrm{a}^{*} \mathrm{~b}^{*}$ plane. For display purposes we used the average among all samples and measurement times $\mathrm{L}^{*}=42.5$ to plot the boundaries between raspberry and strawberry and between strawberry and redcurrant. The boundary between redcurrant and salmon was 
plotted at $\mathrm{L}^{*}=46.1$ because it is the approximate $\mathrm{L}^{*}$ value of the two samples that effectively cross this boundary.

As seen in Fig. 3, all samples follow a similar colour evolution, having an initial raspberry colour that evolves to strawberry and then to redcurrant. After 16 months, two samples (s1 and s3) attained the salmon colour while the rest of samples were still catalogued as redcurrant. Using the time evolution models we also calculated the time that each sample spent in each colour category. Fig. 4 shows that each wine sample evolved at its own pace, although similarities could also be observed between them. All samples, except sample $\mathrm{s} 5$, retained a raspberry colour up to three to four months from the first measurement (until July or August). After seven to eight months (November to December) all were still in the strawberry category. Further evolution depended on the sample and samples $\mathrm{s} 1$ and $\mathrm{s} 3$ reached the salmon colour category by the end of the experiment while the rest of the samples retained redcurrant colour. Evolution models are valid only within the studied period of time and extrapolations beyond the 16-month
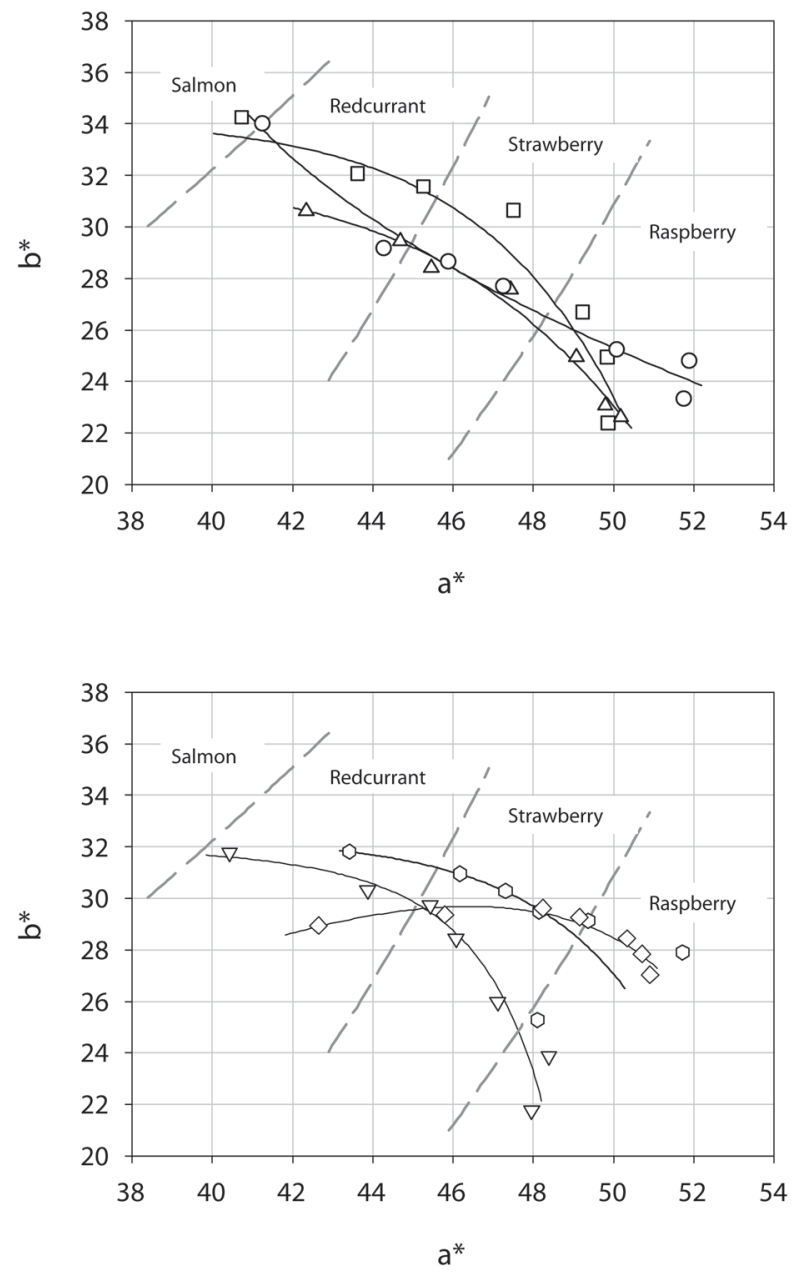

FIGURE 3

Sample colour evolution in the CIELAB $a^{*} b^{*}$ plane. Each symbol corresponds to a different sample (s1: circles; s2: up triangles; s3: squares; s4: diamonds, s5: down triangles, s6: hexagons). Solid lines are the model predictions. Dashed lines represent the boundaries between visual colour categories obtained from a previous study in rosé wines with similar characteristics. period must be done with caution. Sample s5, for instance, appeared to be very close to the redcurrant-salmon boundary in the last measurement. However, the model predicted that this sample would cross the boundary at $t=21.5$ months after the first measurement, i.e. about half a year after the last measurement. For the rest of the samples, boundary crossing predictions were even longer and not reliable.

Wine samples s1, s5 and s6 were bottled with natural cork closures while used synthetic closures were used for samples s2, s3 and s4. Comparison between the two groups did not evidence influence of the type of closure in the colour evolution of the samples. If some effect in this direction exists, it must be hidden in the intrinsic differences between our samples due to grape varieties or winemaking techniques.

\section{CONCLUSIONS}

We studied the colour evolution of a representative set of rosé wines during the first sixteen months after bottling. The studied period of time exceeded the one-year interval between commercialisation of consecutive vintages and it is comparable to the lifetime of this product.

The colour measurement method reproduced the observing conditions during wine visual colour assessment regarding wine sampler, illuminating source, observing background and sample-observer geometry. Measurements provided CIELAB colour coordinate values that can be used to test and compare different time dependence models in the colour evolution of each sample. CIELAB $\Delta \mathrm{E}^{*}$ colour differences between measured and predicted colour are less than human colour discrimination thresholds and model predictions therefore are accurate for any practical application related to the visual colour appearance of this product.

This measurement procedure did not require any sample preparation and it is simpler to implement, compared to transmittance measurements. Furthermore, it is well related to the traditional classification of wine colour into colour categories and is accurate for studying the time evolution of the

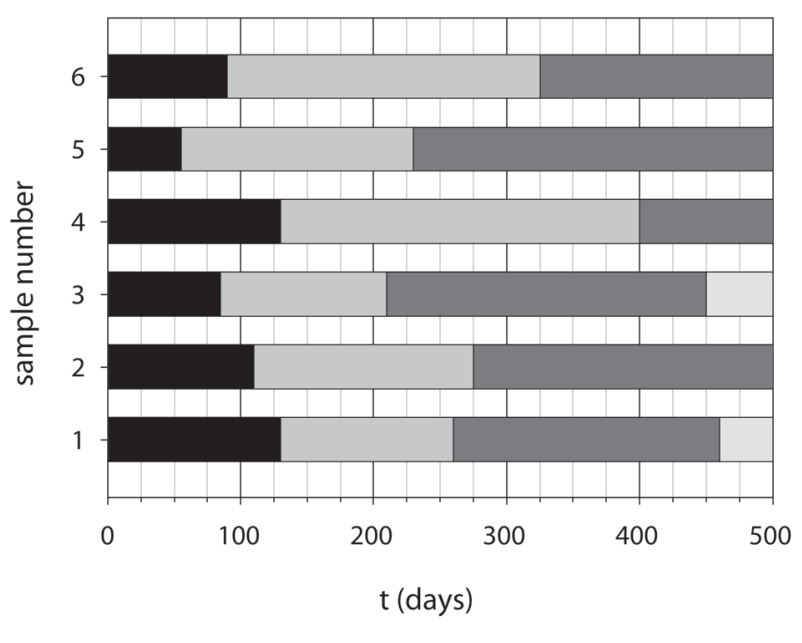

FIGURE 4

Classification into colour categories as a function of time deduced from the time evolution models.

(raspberry: black, strawberry: medium gray, redcurrant: dark gray, salmon: light gray). 
colour characteristics of each sample.

Transmission methods provide objective colour parameters for studying the colour evolution and colour stability of wine samples. However, the proposed methodology is closer and better related to the visual experience during wine tasting by expert tasters and consumers.

\section{LITERATURE CITED}

Alcalde-Eon, C., Escribano-Bailon, M.T., Santos-Buelga, C. \& Rivas-Gonzalo, J.C., 2006. Changes in the detailed pigment composition of red wine during maturity and ageing - A comprehensive study. Anal. Chim. Acta 563(1-2), 238254.

Bartowsky, E.J., Costello, P.J., Villa, A. \& Henschke, P.A., 2004. The chemical and sensorial effects of lysozyme addition to red and white wines over six months' cellar storage. Aust. J. Grape Wine Res. 10(2), 143-150.

CIE, 2004. Colorimetry ( $3^{\text {rd }}$ ed). Vienna, Commission Internationale de l'Eclairage.

Gawel, R. \& Godden, P.W., 2008. Evaluation of the consistency of wine quality assessments from expert wine tasters. Aust. J. Grape Wine Res. 14(1), 1-8.

Glories, Y., 1984. Le coleur des vins rouges, 2e partie: Mesure, origine et interpretation. Connaissance de la Vigne et du Vin 18, 253-271.

Gomez-Miguez, M.J., Gomez-Miguez, M., Vicario, I.M. \& Heredia, F.J., 2007. Assessment of colour and aroma in white wines vinifications: Effects of grape maturity and soil type. J. Food Engin. 79(3), 758-764.

Gomez-Miguez, M.J., Gonzalez-Miret, M.L., Hernanz, D., Fernandez, M., Vicario, I.M. \& Heredia, F.J., 2007. Effects of prefermentative skin contact conditions on colour and phenolic content of white wines. J. Food Engin. 78(1), 238-245.

Hernández, B., Sáenz, C., Alberdi, C., Alfonso, S., Diñeiro, J.M., Fernández de la Hoz, J. \& De Santiago, J.V., 2008. Wine colour vocabulary: What does garnet, ruby, strawberry or straw yellow mean in a colour system? Interim Meeting of the International Colour Association AIC 2008, "Colour Effects and Affects", Stockholm (Sweden).

Hernández, B., Sáenz, C., Fernández de la Hoz, J., Alberdi, C., Alfonso, S. \& Diñeiro, J.M., 2009. Assessing the color of red wine like a taster's eye. Color Res Applic. 34(2):, 153-162.

Huertas, R., Yebra, A., Perez, M.M., Melgosa, M. \& Negueruela, A.I., 2003. Color variability for a wine sample poured into a standard glass wine sampler. Color Res. Applic. 28(6), 473-479.

Jackson, R.S., 1994. Wine science: Principles and applications. San Diego; London, Academic Press.

Jackson, R.S., 2002. Wine tasting: A professional handbook. San Diego, Academic Press.

Little, A.C., 1980. Colorimetry of Wines. Color Res. Applic. 5(1), 51-56.

Maga, J.A., 1974. Influence of color on taste thresholds. Chem. Senses and Flavour 1(1), 115-119.

Mahy, M., Van Eycken, L. \& Oosterlinck, A., 1994. Evaluation of uniform color spaces developed after the adoption of CIELAB and CIELUV. Color Res. Applic. 19(2), 105-121.
Martinez, J.A., Melgosa, M., Perez, M.M., Hita, E. \& Negueruela, A.I., 2001. Note. Visual and instrumental color evaluation in red wines. Food Sci. Technol. Int. 7(5), 439-444.

O.I.V., 1978. Recueil des méthodes internacionales d'analyse des vins. Office Internacional de la Vigne et du vin, Paris.

Ough, C.S. \& Amerine, M.A., 1967. Rose wine color preference and preference stability by an experienced and an inexperienced panel. J. Food Sci. 32(6), 706711

Parr, W.V., White, K.G. \& Heatherbell, D.A., 2003. The nose knows: Influence of colour on perception of wine aroma. J. Wine Res. 14(2), 79-101.

Peng, Z., Duncan, B., Pocock, K.F. \& Sefton, M.A., 1998. The effect of ascorbic acid on oxidative browning of white wines and model wines. Aust. J. Grape Wine Res. 4(3), 127-135.

Pridmore, R.W., Huertas, R., Melgosa, M. \& Negueruela, A.I., 2005. Discussion on perceived and measured wine color. Color Res. Applic. 30(2), 146-149.

Recamales, A.F., Sayago, A., Gonzalez-Miret, M.L. \& Hernanz, D., 2006. The effect of time and storage conditions on the phenolic composition and colour of white wine. Food Res. Int. 39(2), 220-229.

Ribéreau-Gayon, P., 2000. Other winemaking methods. Handbook of enology, vol 1. The Microbiology of Wine and Vinifications. Chichester; New York, Wiley.

Saenz Gamasa, C., Hernandez, B., Santiago, J.V. de, Alberdi, C., Alfonso, S. \& Dineiro, J. M., 2009. Measurement of the colour of white and rose wines in visual tasting conditions. Eur. Food Res. Technol. 229(2), 263-276.

Salinas, M.R., Garijo, J., Pardo, F., Zalacain, A. \& Alonso, G.L., 2003. Color, polyphenol, and aroma compounds in rose wines after prefermentative maceration and enzymatic treatments. Am. J. Enol. Vitic. 54(3), 195-202.

Schanda, J., 2007. Colorimetry: Understanding the CIE system. Hoboken, N.J., Wiley; Chichester: John Wiley.

Skouroumounis, G.K., Kwiatkowski, M., Sefton, M.A., Gawel, R. \& Waters, E.J., 2003. In situ measurement of white wine absorbance in clear and in coloured bottles using a modified laboratory spectrophotometer. Aust. J. Grape Wine Res. 9(2), 138-147.

Skouroumounis, G.K., Kwiatkowski, M.J., Francis, I.L., Oakey, H., Capone, D.L., Peng, Z., Duncan, B., Sefton, M.A. \& Waters, E.J., 2005. The influence of ascorbic acid on the composition, colour and flavour properties of a Riesling and a wooded Chardonnay wine during five years' storage. Aust. J. Grape Wine Res. 11(3), 355-368.

Versari, A., Boulton, R.B. \& Parpinello, G.P., 2008. A comparison of analytical methods for measuring the color components of red wines. Food Chem. 106(1), 397-402.

Williams, A.A., Langron, S.P. \& Noble, A.C., 1984. Influence of appearance on the assessment of aroma in Bordeaux wines by trained assessors. J. Inst. Brew. 90(4), 250-253.

Wyszecki, G. \& Stiles, W.S., 2000. Color science: Concepts and methods, quantitative data, and formulae. New York, John Wiley. 